Pour une relecture matérialiste de la forme scolaire

\title{
Matérialisme et révolution chez Célestin Freinet
}

\section{Antoine Janvier}

URL : http://journals.openedition.org/grm/1537

DOI : $10.4000 / \mathrm{grm} .1537$

ISSN : $1775-3902$

Éditeur

Groupe de Recherches Matérialistes

\section{Référence électronique}

Antoine Janvier, « Matérialisme et révolution chez Célestin Freinet », Cahiers du GRM [En ligne], 14 |

2019, mis en ligne le 13 juin 2019, consulté le 10 décembre 2020. URL : http://

journals.openedition.org/grm/1537 ; DOI : https://doi.org/10.4000/grm.1537

Ce document a été généré automatiquement le 10 décembre 2020.

(c) GRM - Association 


\title{
Matérialisme et révolution chez Célestin Freinet
}

\author{
Antoine Janvier
}

\section{NOTE DE L'AUTEUR}

Cet exposé a été préparé par des ateliers de lecture de textes de Freinet dans le cadre d'un séminaire de philosophie de l'éducation organisé à l'Université de Liège en 2017-2018, avec Emmanuel Chapeau, Aurore Compère, Grégory Cormann, Caroline Glorie et Anne Herla. Il doit donc beaucoup à leurs remarques, suggestions, et aux échanges dont il s'est nourri.

1

1 Le nom de Freinet est, depuis une trentaine d'années au moins, associé à ce qu'on appelle, d'expressions variables et flottantes, la " pédagogie nouvelle » ou la " pédagogie active », au point que c'est sans doute à ce qu'on appelle « la pédagogie Freinet » que l'on pense de prime abord quand on évoque les pédagogies nouvelles ou les pédagogies actives.

Ce texte vise à compliquer cette évidence, et à donner une image différente de l'expérience, ou des expériences entreprises par Freinet, en mettant l'accent sur deux dimensions du projet théorique et pratique de Freinet, matérialisme et révolution, qui le singularisent dans le paysage des pédagogies nouvelles. Une telle perspective suppose de considérer le travail de Célestin Freinet comme un travail mené en situation (au sens de Sartre), et par conséquent de le considérer en train de se faire, dans un débat permanent avec la conjoncture théorique et pratique dans laquelle il s'élabore, sans présupposer certains de ses résultats comme évidents ou donnés d'emblée. C'est pourquoi je ne traiterai pas de «la» pédagogie Freinet «en général», ou plutôt sur un mode systématique, comme si les "grands» textes de Freinet (si du moins il en est), pour l'essentiel publiés dans l'immédiat après-guerre (Essai de psychologie sensible, L'école 
moderne), disaient au fond la même chose et mieux et avec plus d'assurance, de précision et de rigueur, ce que le jeune Freinet pensait déjà dans les années 1920 en arrivant comme instituteur dans la petite école de Bar-sur-Loup : comme si, au fond, Freinet avait toujours déjà été Freinet, et que l'imprimerie à l'école, le fichier scolaire coopératif, le texte libre, n'avaient été que des «innovations pédagogiques » introduites de l'extérieur dans une école vieillie par un Freinet qui les auraient toujours déjà conçues, ou mieux, toujours déjà " possédées ", fût-ce inconsciemment, génial pédagogue n'ayant au fond rien d'autre à faire qu'à se découvrir lui-même et à mûrir au fil des années, dans un milieu plus ou moins hostile, plus ou moins favorable ou défavorable à la pleine expression de ses potentialités - comme le bon vin.

3 A rebours de cette tendance qui manie allègrement l'illusion rétrospective, je tenterai ici de reprendre la lecture de Freinet au point de départ, en me penchant sur ses premiers textes, ceux des années 1920 : ces textes dans lesquels Freinet conquiert sa propre pensée et rend compte de ses premières tentatives, des tentatives au cours desquelles il découvre pour son compte ses propres innovations pédagogiques. C'est à cette condition que nous pourrons ressaisir la nécessité interne de la recherche de Freinet, celle qui, précisément, le conduit à expérimenter autre chose non seulement que ce que l'institution scolaire de la III ${ }^{e}$ République lui propose, mais aussi autre chose que ce que les pédagogies nouvelles elles-mêmes proposent en contrepoint. On verra que la singularité de la recherche menée par Freinet tient autant, sinon plus, à l'insatisfaction qu'il éprouve face aux pédagogies nouvelles qui connaissent un succès et une coordination sans précédent au début des années 1920, qu'à l'insuffisance de la pédagogie scolastique de l'école de Jules Ferry - si du moins elle existe. Et mon hypothèse, c'est que cette insatisfaction est profondément politique, et qu'elle s'exprime dans ces deux enjeux par lesquels il lui arrive de réfléchir et de nommer son projet : matérialisme et révolution. Le travail pédagogique de Freinet est en somme un travail politique, parce qu'il se veut matérialiste et révolutionnaire.

\section{2}

4 Si l'expression d'«école nouvelle " ou d'« éducation nouvelle» rencontre dès le XIX siècle un succès certain (notamment sous la plume et l'expérience entreprise par Edmond Demolins à l'Ecole des Roches $\left.{ }^{1}\right)$, c'est au début du XXe siècle, et plus encore après la première guerre mondiale qu'elle fait véritablement fortune, confondue avec celle d'éducation "active». La figure centrale de ce qui devient alors un mouvement international est le suisse Adolphe Ferrière. Ferrière publie de nombreux ouvrages destinés à faire connaître les écoles nouvelles et les principes et méthodes de l'éducation active, centrée sur l'enfant, son rythme de développement, ses intérêts et sa spontanéité. Mais il est surtout le grand " coordinateur » des différentes expérimentations menées à l'échelle européenne et mondiale : Ferrière fonde en effet le Bureau international des écoles nouvelles en 1899 (BIEN) $^{2}$ puis, avec d'autres, la Ligue internationale pour l'éducation nouvelle en 1921 (LIEN) $)^{3}$, qui rassemble de grandes figures comme l'italienne Maria Montessori, le belge Ovide Decroly, les suisses Edouard Claparède et Jean Piaget, les anglais Beatrice Ensor et Alexander Neill, le français Roger Cousinet, l'américain John Dewey, Elisabeth Rotten pour l'Allemagne... Outre ses congrès annuels, la Ligue publie une revue internationale à partir de 1922, Pour l'ère nouvelle $e^{4}$, à la lecture de laquelle on peut mesurer l'ambition « internationaliste » de la Ligue, et la centralité de la psychologie de l'enfant, j'y reviendrai, qui soutient l'essor du mouvement d'éducation nouvelle. 
5 A l'été 1923, la LIEN tient son deuxième Congrès, à Montreux (Suisse). Membre de la Ligue, Freinet parvient à y assister. Depuis 1920, il est instituteur adjoint dans une école rurale, à Bar-sur-Loup, dans les Alpes-Maritimes. Gravement blessé au poumon en octobre 1917, sur le front à la bataille de Malmaison qui fait suite à l'échec essuyé par l'armée française au Chemin des dames au printemps de la même année ${ }^{5}$, Freinet est amputé de ses capacités respiratoires ${ }^{6}$ et se retrouve à Bar-sur-Loup, à 23 ans, dans l'impossibilité d'assurer ses leçons de manière traditionnelle, mais également convaincu que l'éducation et l'institution scolaire ne sont pas innocentes dans la boucherie de 14-18. C'est pourquoi il se met à la recherche de pratiques pédagogiques différentes, à la fois plus en phase avec ses capacités physiques, et plus adéquates à son humanisme pacifiste et anti-nationaliste. On sait qu'il lit avidement Ferrière, Montessori, Decroly, Dewey... C'est ainsi qu'il se rend au Congrès de Montreux en 1923.

Dans le récit publié en 1947 et réédité chez Maspero en 1968 qu'elle consacre à la Naissance d'une pédagogie populaire, Elise Freinet, l'épouse de Célestin, raconte le paradoxal désarroi éprouvé par le petit instituteur d'école rurale aux prises avec la population pauvre de la campagne face aux enthousiasmantes grandes entreprises des écoles nouvelles :

[Freinet] se rend à Montreux, heureux d'avance d'y entendre Ferrière, Claparède et Bovet $^{8}$. Il est passionnément intéressé par tout ce qu'il voit et entend. Il pénètre mieux ce principe d'éducation nouvelle soucieux de donner à l'enfant un rôle actif dans sa propre éducation. Mais à chacune des affirmations et des perspectives que les pédagogues de Genève imposent à son esprit, il est d'avance découragé. Il se rend compte qu'il y a une éducation nouvelle relativement facile, applicable pour les écoles possédant le matériel éducatif, l'installation scolaire permettant l'activité de l'enfant et l'individualisation de l'enseignement. Mais pour l'école de Bar-surLoup le problème est tout autre. L'image de sa petite classe dénudée et poussiéreuse s'impose à lui et lui serre le coeur.

[...]

Sa tristesse et sa solitude dans ce Congrès où de grands éducateurs affirment déjà tant de victoire en face de ses hésitations ont au moins l'avantage de le rejeter farouchement vers ce matérialisme scolaire qui reste son plus noble souci [...]. Il prend conscience plus encore de la dépendance étroite de l'école et du milieu, et combien la société conditionne l'école et l'enseignement.

Il n'y a pas de pédagogie sans que soient remplies les conditions économiques favorables permettant l'expérience et la recherche. Il n'y a pas d'éducation idéale, il n'y a que des éducations de classes.

7 A bien des égards, ce texte d'Elise Freinet est essentiel. Mais il ne faut pas l'interpréter trop vite.

\section{3}

On pourrait en effet en conclure simplement que ce qui distingue Freinet des célèbres pédagogues de la LIEN, ce sont les conditions socio-économiques dans lesquelles ils développent leurs expérimentations pédagogiques, et plus encore, la prise en compte ou non de ces conditions dans la recherche pédagogique. Il est vrai que c'est dans ce sens que Célestin Freinet lui-même rendra compte de ce Congrès de Montreux dans un article rédigé pour la revue d'Henri Barbusse, Clarté, en novembre 1923. Le texte est des plus sévères. Tout en se terminant par l'idée qu'il faut étudier les écoles nouvelles «parce qu'elles sont [...] les laboratoires de la Science de l'Education », et s'il présente en ce sens 
une série d'écoles nouvelles (Ecole des Roches, Ecole de Faria de Vasconcellos à Bruxelles, libre communauté scolaire d'Odenwald, Ecole "jardin " pour filles de Londres, etc.), il met l'accent sur le coût de ces établissements (frais d'écolage à 15000 francs par an et besoins en personnel à hauteur de " 2 à 4 élèves par maître ») qui rend impossible toute perspective de généralisation populaires de telles formes d'enseignement. Et surtout, dans une prose ironique et acerbe («Congrès honnête, académique, où l'on écoute sans passion, où l'on discute à peine [...]. On a même entendu à Montreux M. Emile Coué, le chef du mouvement actuel pour l'auto-suggestion, qui nous invita à dire sagement matin et soir la nouvelle prière par lui inventée, tout en dévidant vingt grains de notre chapelet.»), ce texte dégage la perspective bourgeoise qui gouverne ces initiatives, incapable d'affronter les « relations entre l'Ecole et la société »- et quand, par exception, c'est le cas, c'est sous la forme d'un espoir naïf et idéaliste, celui de "persuader les pouvoirs publics de la portée sociale et humaine d'une bonne éducation. $»^{9}$

Cette critique, Freinet ne la mène pas seulement depuis sa position singulière de petit instituteur en proie à la misère rurale. Comme Grégory Chambat a eu le mérite de le rappeler et de le mettre en évidence dans les publications récentes qu'il a consacrées à Freinet, l'entrée de Freinet en pédagogie se fait d'abord et avant tout sur le terrain syndical - dès son arrivée à Bar-sur-Loup ${ }^{10}$. Un terrain double. D'une part, Freinet est membre de la Fédération des syndicats des membres de l'enseignement laïque (FSMEL), seule fédération nationale de la CGT à ne pas sacrifier à l'Union sacrée pendant 14-18. Au moment de la scission de 1921 entre réformistes (CGT) et révolutionnaires (CGT-U), la FSMEL prend le parti révolutionnaire, tout en y faisant figure de minorité singulière, gardant une tradition anarcho-syndicaliste face à l'alignement sur le centralisme démocratique de la Troisième Internationale et la subordination au Parti. ${ }^{11}$ Durant les années 1920, Freinet écrira de très nombreux articles pour la revue de la FSMEL, L'Ecole émancipée. D'autre part, Freinet est envoyé par son syndicat au Congrès de Bordeaux, à l'été 1920, où il participe à la fondation de l'Internationale des travailleurs de l'enseignement, qu'il présente comme "une organisation de classe dont l'action, parallèle à celle des groupements ouvriers, se développe dans le plan révolutionnaire. $»^{12}$ C'est via cette structure que, à l'été 1925, Freinet fera un voyage en URSS et y découvrira les expériences en matière scolaire favorisées par les bolchéviques - ou, du moins, qu'ils n'empêchent pas encore de se développer.

10 L'engagement de Freinet doit beaucoup, sans doute, à son expérience de guerre ${ }^{13}$. Ce qui est frappant cependant, c'est la tournure qu'il lui confère d'emblée, et qui s'indique à travers une formule: pour la révolution à l'école. Freinet refuse de concevoir l'engagement syndical révolutionnaire comme un engagement qui concernerait le syndicaliste Freinet et non l'instituteur, un engagement purement social et politique. C'est sans doute là l'héritage de la position du mouvement l'Ecole Emancipée depuis sa création dans les années 1910 ; mais il n'est pas exagéré de dire que, dans ces années 1920, Freinet donne toute son ampleur et sa radicalité à cette position.

\section{4}

Dans un article publié dès le mois d'octobre 1920 dans L'Ecole émancipée et qui présente l' Internationale des travailleurs de l'enseignement sous le titre "Pour la révolution à l'école ", Freinet écrit avec son camarade allemand Heinrich Siems : 
Notre Congrès de Bordeaux a été avant tout un congrès politique, très intéressant, certes, et peut-être nécessaire. Mais nous n'avons pas su y montrer que nous étions instituteurs. Nous nous sommes posés en syndicalistes révolutionnaires, mais jamais en instituteurs révolutionnaires.

Il ne suffit donc pas de militer à côté du travail effectué en classe sur le plan pédagogique pour la transformation communiste ou socialiste de la société et, avec elle, pour une transformation de l'école qui permettrait, par exemple, la généralisation «prolétarienne » des méthodes nouvelles aujourd'hui réservées à quelques membres de l'élite bourgeoise ou à quelques défavorisés privilégiés par le regard bienveillant d'un pédagogue philanthrope. C'est pourquoi Freinet refuse tout autant la posture « attentiste » sur le plan pédagogique au nom du travail syndicaliste-révolutionnaire, que la posture " pédagogiste bourgeoise » qu'il reprochait d'emblée aux grandes figures de la LIEN. Se contenter d'attendre que la « révolution soit faite " pour escompter que l'école et la pédagogie s'en trouveront par conséquent nécessairement transformées pose problème : car, remarque Freinet, par

la «Révolution faite « on n'entend souvent que la transformation politique qui, selon Lénine lui-même, ne sera que le début d'une longue période qui peut durer plusieurs générations - pendant lesquelles il faudra une dictature plus ou moins dure pour diriger des hommes qui seront incapables encore d'être libres. Devonsnous admettre que notre pédagogie - même au lendemain de la Révolution - doive s'incliner devant un ordre nouveau, qui par la force des choses, aura beaucoup d'analogies avec l'ordre actuel? Et, négligeant notre vrai devoir - éclairer les esprits - deviendrons-nous les soutiens d'un dogme nouveau qui sera pourtant loin encore d'être le communisme ? ${ }^{14}$

13 Prendre dès maintenant une attitude révolutionnaire à l'égard de la pédagogie ellemême, c'est-à-dire des formes d'apprentissage et de développement de l'enfant et des types de rapports sociaux au sein de la classe, c'est s'attaquer aux modalités spécifiquement scolaires et pédagogiques de la domination imposée par la société capitaliste, qui ne manqueraient pas, sinon, de faire brutalement ou insidieusement retour au moment de la transition révolutionnaire et de compromettre l'avènement du communisme.

Et si toutes les foules affamées se dressaient enfin et qu'on les mène à la Révolution, que sera notre école au lendemain de cette action? Une grosse majorité d'instituteurs antirévolutionnaires dont quelques-uns sauront résister, n'en doutez pas. Et puis improvisez-vous notre organisation future ? Et les livres de classe ? Et l'éducation des masses dans les villages surtout? Oui, nous ferons la Révolution chez les autres, mais, après la libération, nous ferons encore tous à nos enfants oh! ne récriminez pas! - des âmes d'esclaves. Et nous qui avons la charge de faire des hommes nouveaux, nous ne saurons produire que des contre-révolutionnaires, ou des égarés qui se livreront aussitôt à quelque nouveau Napoléon. ${ }^{15}$

Le « matérialisme » de Célestin Freinet tient très exactement à cet effort d'articulation du plan pédagogique et du plan socio-politique, sans sacrifier l'un à l'autre. Car un tel effort ne signifie rien d'autre que prendre acte d'une double impossibilité : l'impossibilité d'une éducation idéale dans les conditions actuelles (pour reprendre la formule d'Elise Freinet: « il n'y a pas d'éducation idéale »), et l'impossibilité de ne pas oeuvrer à la transformation de l'école quand on milite pour la cause prolétarienne. Et c'est précisément l'inscription de sa recherche politico-pédagogique dans l'affrontement lucide de cette double impossibilité qui fait de Freinet un pédagogue matérialiste. 
15 Si Freinet se revendique volontiers "pédagogue révolutionnaire ", il ne fait guère usage pour son compte du qualificatif de "matérialiste ", bien qu'il puisse lui arriver de s'en servir, de manière occasionnelle et, il faut bien le dire, assez lâche (Freinet n'est pas un homme de concepts). ${ }^{16}$ C'est Elise Freinet qui a mis l'accent sur cette dimension de la pensée de Freinet, en parlant précisément de "matérialisme scolaire $»^{17}$. Que faut-il entendre par là? A ma connaissance, cette question n'a guère soulevé l'enthousiasme ni suscité d'analyse serrée de la part des commentateurs - moins encore que l'ambition « révolutionnaire » et « sociale », ou « populaire », du projet pédagogique de Freinet.

\section{6}

Dans ses Cinq études sur Célestin Freinet, Alain Vergnioux définit rapidement, en passant, «le matérialisme de Freinet» comme « une certaine conception du travail social, actif, producteur, s'appuyant sur des outils, impliquant l'entraide et la solidarité des travailleurs $\gg .{ }^{18}$ C'est bien l'évidence: Freinet est matérialiste en vertu d'une anthropologie héritière de Marx selon laquelle l'homme est un être de travail et réalisera la totalité de ses potentialités à travers le travail, et en vertu de la stricte application de cette anthropologie en pédagogie. Vergnioux écrit en effet que, pour Freinet, «l'école de demain [...] sera "l'école du travail", le travail étant perçu dans la perspective du matérialisme marxiste comme facteur d'émancipation. $»^{19}$

17 Il est vrai que Freinet accorde une grande part à la question du travail à l'école; mais précisément comme une question, ou un problème, étant donnée la situation de l'école en régime capitaliste. Ainsi, dans un article de 1924 pour la revue Clarté, Freinet peut-il louer l'effort des écoles nouvelles allemandes qui ont développé des pratiques éducatives basées sur le travail manuel, mais en indiquant tout de même une sérieuse réserve : elles restent animées d'une " conception petite-bourgeoise et réformiste de l'Ecole nouvelle ", au sens «où le travail n'est qu'un moyen pour faciliter l'acquisition et la culture capitalistes. $»^{20}$ Dans un autre texte, toujours publié dans Clarté, Freinet soutient que « les humbles artisans de l'Ecole du Travail allemande » furent contraints de

composer avec les idées de leurs maîtres. Il fallut prouver à ceux-ci que le travail manuel, aux champs et à l'atelier, facilite au lieu d'entraver l'acquisition des connaissances, que l'élève s'instruit d'avantage en réalisant manuellement ce qu'on a voulu jusqu'ici lui apprendre verbalement: bref, que le travail, tel qu'il est introduit dans ces écoles, est un adjuvant précieux, une «illustration » nécessaire des belles leçons. ${ }^{21}$

18 Cette réserve, Freinet la manifeste également à l'égard des écoles communautaires de Hambourg qui, initiées pendant la conjoncture révolutionnaire allemande de 1918, se sont maintenues tant bien que mal par delà son échec ${ }^{22}$.

C'est que, quelle que soit la valeur professionnelle et l'esprit pédagogique des maîtres, ces écoles sont impuissantes à se maintenir hors de leur élément qui est la Révolution. Non pas tant qu'elles aient à redouter l'hostilité déclarée de la population ou des pouvoirs publics dans une ville comme Hambourg où l'élément ouvrier est tout de même puissant - témoin le récent soulèvement communiste. Mais c'est tout l'esprit capitaliste qui se ligue inévitablement contre une discipline révolutionnaire. ${ }^{23}$ 
19 En réalité, jamais Freinet ne se satisfait de la perspective d'une école reconstruite sur les bases d'une organisation productive et coopérative du travail, et ce n'est pas dans un tel espoir que l'on trouvera l'expression de son matérialisme. Car, pour Freinet, une telle perspective ne peut être envisagée de manière abstraite: elle doit être considérée en fonction des conditions données du régime socio-économico-politique dans lesquelles elle peut être réalisée ${ }^{24}$.

\section{7}

Quand Elise Freinet évoque le matérialisme scolaire de Freinet, c'est pour mettre en évidence l'extrême attention que Freinet porte à ce qui résiste à toute idéalisation d'une expérience de transformation. Rappelons-nous ce texte où il est question du sentiment de Freinet au Congrès de la Ligue internationale pour l'éducation nouvelle de Montreux :

Sa tristesse et sa solitude dans ce Congrès où de grands éducateurs affirment déjà tant de victoire en face de ses hésitations ont au moins l'avantage de le rejeter farouchement vers ce matérialisme scolaire qui reste son plus noble souci [...]. Il prend conscience plus encore de la dépendance étroite de l'école et du milieu, et combien la société conditionne l'école et l'enseignement.

21 Le matérialisme de Freinet ne renvoie pas à un élément de doctrine, au contenu d'une théorie, mais à une attitude : c'est d'abord une attitude qui consiste à partir de ce qui est là , et avec lequel il faut bien faire quelque chose, plutôt qu'à fantasmer sa suspension ou son annulation. Ce qui est là, c'est par exemple l'école publique, en milieu rural, avec son manque de moyens matériels (Freinet s'en plaint à longueur de correspondance) et ses élèves de toutes sortes. Ce qui est là, c'est aussi, plus largement, le régime capitaliste dont on ne peut faire abstraction, ni changer d'un claquement de doigt. Tel est précisément ce que, fondamentalement, Freinet critique dans les ambitions « politiques » qui se profilent derrière le mouvement d'éducation nouvelle.

Nous ne nous faisons [...] pas d'illusions : nous ne pensons pas, comme nombre de purs pédagogues suisses ou allemands, que l'école peut régénérer le monde, qu'elle peut préparer la paix et l'union des peuples, qu'elle peut amener le triomphe du travail. Nous tous, qui sommes mêlés chaque jour à la dure lutte de l'école contre le milieu social, savons bien que ce n'est là qu'un beau rêve. L'éducation maximum que nous pouvons donner sera vite anéantie par les influences abrutissantes de la presse, du cinéma, de l'organisme capitaliste tout entier. ${ }^{25}$

C'est que, croire que l'on peut régénérer le monde par l'éducation scolaire, c'est annuler magiquement, par l'imagination, le monde pour le recréer dans un avenir utopique qui dispense ainsi de s'affronter au monde tel qu'il est donné, et donc d'œuvrer à sa transformation. On retrouve ici, dans cette critique de Freinet, quelque chose de la critique que Marx adressait - à tort ou à raison, ce n'est pas notre affaire en l'occurrence - à ce qu'il appelait dans le Manifeste «le socialisme et le communismes utopiques et critiques ». On rappellera ces méchancetés brièvement, pour éclairer la position de Freinet : c'est leur impuissance politique, qui tient selon Marx à « ce que faisaient défaut les conditions matérielles » de l'émancipation du prolétariat, qui conduit « les inventeurs de ces systèmes ", à se mettre " en quête d'une science sociale, de lois sociales, à seule fin de créer ces conditions ", mais de les créer fantasmatiquement :

Leur ingénieuse activité personnelle doit se substituer à l'activité sociale; ils veulent remplacer les conditions historiques de l'émancipation par des conditions tirées de leur imagination, et l'organisation réelle et graduelle du prolétariat en 
classe sociale par une organisation spécialement élucubrée de la société. L'histoire future se résout pour eux dans la propagande et la mise en pratique de leurs plans de société.

En forgeant leurs plans, ils ont, sans doute, conscience de défendre avant tout l'intérêt de la classe la plus misérable, de la classe laborieuse. Et c'est sous ce seul aspect de la souffrance extrême que le prolétariat existe pour eux.

La forme rudimentaire de la lutte de classes tout comme leur propre situation dans la vie les portent cependant à se croire au-dessus des oppositions de classes. Ils voudraient améliorer l'existence de tous les membres de la société, même les plus privilégiés. C'est pourquoi ils lancent sans cesse leur appel à l'ensemble de la société sans distinction, et même de préférence à la classe dominante. Ne suffit-il pas de comprendre leur système, pour reconnaître qu'il offre le meilleur de tous les plans de la meilleure des sociétés possibles?

C'est pourquoi ils rejettent toute action politique, et surtout toute action révolutionnaire. Ils veulent atteindre leur but par des moyens pacifiques, et ils essaient de frayer un chemin au nouvel évangile social par la force de l'exemple, par des expériences limitées, qui naturellement, se terminent par un échec. ${ }^{26}$

Freinet ne cite guère Marx - il évoque plus volontiers Lénine. Mais l'analogie (je n'ai pas dit: la comparaison) est frappante. Freinet ne cesse d'insister sur le double geste d'abstraction à l'égard d'un milieu social défavorable et de sur-personnalisation que supposent les écoles nouvelles en Europe et aux Etats-Unis, c'est-à-dire en régime capitaliste, et qui les conduit inévitablement à l'échec, ou plus exactement qui marque d'impossible leur tentative :

Notre siècle est loin d'être exclusivement théorique en éducation. [...] Et cependant ces réalisation ne sont ni bien nombreuses ni suffisamment concluantes, parce qu'elles se sont heurtées, le plus souvent, à l'esprit capitaliste, qui, alors même qu'il leur semblait favorable, était par son essence un ferment destructeur. Aussi comprend-on que les seules écoles ayant tenté de donner une éducation individuelle et sociale rationnelle se soient installées à l'écart d'une société dont elles craignent l'influence. ${ }^{27}$

L'erreur de quelques pédagogues occidentaux est justement de croire possible l'instauration chez nous d'une autre école. Ils ne se rendent pas compte par quel artifice, ou plutôt par quels prodigieux efforts de tous les instants leurs écoles nouvelles vivotent et travaillent; et les instituteurs eux-mêmes qui prétendent apporter dans leur classe quelque chose de nouveau n'y arrivent que par une activité personnelle incessante. ${ }^{28}$

On mesurera la force de cette position matérialiste dans le récit que donne Freinet de son voyage en URSS en 1925.

\section{8}

Dès les années 1920, s'informant comme il peut de la situation russe, Freinet en rend compte autant que possible dans la revue Clarté comme du « mouvement éducatif (...) le plus intéressant $»^{29}$, du pays où se sont créées des écoles communautaires « en tous points semblables à l'école à l'Odenwald ou aux écoles de Hambourg ", mais qui "vivent et se développent remarquablement, distançant - au point de vue technique - tout ce qui a été fait dans vieille Europe ", au point d'affirmer que «la Communauté scolaire a enfin trouvé son terrain. ${ }^{30}$

C'est à l'été 1925 que, avec une cinquantaine d'instituteurs, Freinet se rend en URSS. Il participe à un voyage organisé par le Syndicat Panrusse des Travailleurs de l'Enseignement, sous l'égide de l'Internationale des Travailleurs de l'Enseignement. Le 
voyage dure environ un mois, de la fin du mois d'août à la fin du mois de septembre, dont trois semaines passées en Russie. La délégation suivra la visite guidée à la gloire de la politique bolchevique mise en place depuis la Révolution d'octobre, et, en particulier, en matière de politique scolaire. Outre une rencontre avec les responsables de l'éducation publique (Zlata Lilina, Nadia Kroupskaïa, Moisseii Pistrak, et pour la partie de la délégation qui reste quelques jours supplémentaires sur le sol russe, le Commissaire du peuple à l'instruction publique, Lounatcharski), les instituteurs sont invités à visiter des écoles de type divers, mais principalement destinées à l'accueil d'orphelins, vagabonds, ou arriérés mentaux que la révolution et la guerre civile ont laissé derrière elles.

De ce voyage, Freinet donnera un compte-rendu sous forme d'articles publiés sous le titre «Mes impressions de pédagogue en Russie soviétique» de novembre 1925 à juin 1926 dans L'Ecole émancipée (suivra un ouvrage publié en 1927, intitulé Un mois avec les enfants russes). Dans ces articles, Freinet salue avec enthousiasme les expériences pédagogiques qu'il a pu visiter, tout en regrettant que les pédagogues «bourgeois » des écoles nouvelles ne s'y intéressent guère ${ }^{31}$.

La nouvelle école russe a été systématiquement dédaignée par les pédagogues occidentaux parce qu'elle s'intitulait fièrement école du travail et mettait le travail et le matérialisme à la base de la pédagogie. ${ }^{32}$

Voilà revenir le travail. On peut bien sûr, à nouveau, entendre que c'est en ce sens qu'il faut comprendre le "matérialisme" des pédagogues russes: comme le réclame le mouvement communiste depuis au moins la $10^{\mathrm{e}}$ mesure recommandée dans Manifeste de 1848, il faut organiser une " éducation publique et gratuite de tous les enfants » sur le mode d'une «articulation de l'éducation avec la production matérielle». Mais Freinet n'écrit pas que les Russes mettent «le travail ou le matérialisme à la base de la pédagogie "; il écrit qu'ils mettent "le travail et le matérialisme à la base de la pédagogie ». Au risque de surinvestir un peu les formules de Freinet, je dirai qu'ici Freinet ne résume pas le matérialisme des pédagogues russes à la centralité accordée au travail dans l'éducation. Le matérialisme pédagogique implique autre chose qu'une organisation de l'école autour du travail quand bien même cette organisation, en mettant en place un travail libre, socialisé et productif, diffère de l'option social-démocrate allemande qui n'« introduisait le travail manuel à l'école» que pour, au fond, en faire une simple " "méthode" commode pour l'acquisition capitaliste du savoir "33. A quoi renvoie donc le matérialisme des Russes?

On peut le comprendre en se penchant sur la polémique que Freinet entretient dans les pages de L'Ecole Emancipée avec un certain Delaunay, proche d'Adolphe Ferrière et collaborateur de la revue Pour l'ère nouvelle $e^{34}$, une polémique qui entrecoupe la publication du récit de voyage que propose Freinet dans cette même revue, fin 1925 et début 1926. Tout en saluant l'effort pédagogique des Russes, Delaunay met un bémol à l'enthousiasme de Freinet ${ }^{35}$ et lui reproche de nier l'influence des pédagogues "bourgeois » du mouvement d'éducation nouvelle sur les orientations donnés par les russes à la création de leurs écoles nouvelles.

Selon notre camarade les dirigeants de l'instruction publique en Russie ont à peu près tout ignoré des efforts des pédagogues bourgeois. Ceci est tout à fait inexact. La lecture même de leurs programmes nous prouve sans conteste qu'ils connaissaient le plan Dalton [expérience de planification des apprentissages, inspirée par Dewey, qui a eu lieu dans les années 1920 dans la ville de Dalton aux Etats-Unis] et la méthode des projets de Dewey. Il nous paraît certain aussi qu'ils ont connu les travaux du Dr Decroly, tout comme ceux des pédagogues suisses, et 
un article du camarade Lapraz confirme notre opinion : Lounatcharsky, écrit-il, a beaucoup connu à Genève Ferrière et s'est inspiré de ses idées dans son organisation de l'enseignement. Il me semble qu'on ne saurait plus, après cela, faire grief à quelques-uns d'entre nous d'étudier les efforts pédagogiques d'origine bourgeoise. ${ }^{36}$

La réponse de Freinet me semble particulièrement significative. D'une part, il conteste avoir passé sous silence la connaissance que les dirigeants et pédagogues russes ont des pédagogues bourgeois ${ }^{37}$. Mais d'autre part, et surtout, Freinet souligne que cette connaissance ne peut permettre de comprendre le mouvement réel d'expérimentation qui est à l'oeuvre en Russie, en tant qu'il s'agit d'un mouvement de masse qui tient aux besoins et nécessités imposés par la situation révolutionnaire, et qui se caractérise par la multiplicité de ses formes, non-centralisée. Je cite, un peu longuement il est vrai, cette partie de la réponse de Freinet à Delaunay :

"Les nouveaux programmes nous prouvent sans conteste que les Russes connaissent le Plan Dalton » objecte Delaunay. Je l'ai dit moi-même : «On prononce là-bas les mots de Dalton Plan... avec une sorte de vénération ». Il faut bien, dès lors, qu'on connaisse la méthode. Mais je suis heureux de préciser que ce n'est pas par un miracle de méthode seule que le Dalton Plan a acquis, en quelques mois, une telle vogue en Russie. C'est que cette technique de travail était un besoin de la nouvelle pédagogie. Et il ne faut pas croire que ce soit le Dalton Plan qui ait créé l'école libre et travailleuse; c'est au contraire l'école révolutionnaire du travail qui a nécessité et qui a créé le Dalton Plan Russe.

Je regrette aussi de devoir défendre mon erreur en ce qui concerne les travaux de Ferrière, du Dr Decroly et des écoles nouvelles. Je ne sais si Loutnacharsky a beaucoup connu en Suisse M. Ferrière. Si cela est, il n'aura certainement pu que profiter de l'expérience du maître. Mais, même en Russie - et en Russie surtout, dirais-je - ce n'est pas un ministre de l'Instruction publique qui dicte la pédagogie nouvelle. Celle-ci naît, prend forme, devient pratique, dans les écoles expérimentales, puis dans les écoles ordinaires. Et je puis bien affirmer que partout où nous sommes passés, les meilleurs pédagogues qui travaillent dans les écoles expérimentales, les Volinska, les Pistraka; ceux qui sont à la tête de laboratoires de pédagogie dont nous envions l'organisation; les pédagogues même du commissariat de l'Instruction publique ignoraient jusqu'aux noms de Ferrière et de Decroly.

C'est un fait.

Je ne dis qu'il n'y ait, par toute la Russie, quelque éducateur connaissant l'effort des pédagogues d'Europe occidentale. Mais je répète que ceux qui ont le plus travaillé à bâtir la nouvelle pédagogie russe ne connaissaient pas les travaux de Ferrière et de Decroly, et que seule la Révolution, en plaçant l'éducation sur son véritable terrain, en exigeant pour elle des méthodes naturelles et vivantes, a permis à ces pédagogues de s'élever d'emblée à hauteur des pédagogues d'occident et de les dépasser. ${ }^{38}$

L'engouement de Freinet pour la politique scolaire et pédagogique dans la Russie bolchévique peut nous paraitre étrange. Mais les historiens ont montré que les années 1920 occupaient une place à part dans la mise en place de la politique éducative en Russie avant le durcissement des années $1930^{39}$ : aux années d'impulsion de la politique d'alphabétisation, 1918-1920, fortement marquées par les orientations du pouvoir central (mais aussi du coup, par les limites de leur champ d'action) et par la diffusion d'un matériel de propagande qui confondent en un même mouvement éducation et idéologisation des masses, succèdent, avec la fin du communisme de guerre et le tournant de la NEP de 1922, une sorte d'âge d'or de l'expérimentation pédagogique, caractérisée par une « désidéologisation du contenu des textes publiés par les soviets ${ }^{40}$, une vivacité et une multiplicité de la production culturelle, en particulier littéraire, qui se répercutent jusque dans les bibliothèques rurales, et l'installation d'écoles nouvelles (par l'Ecole 
unique du travail) dont « le but pédagogique proclamé est de casser la logique d'autorité et d'inculcation forcée caractéristiques du système éducatif sous le régime tsariste $»^{41}$ :

il n'est pas question d'endoctriner les élèves, de leur faire ingurgiter des manuels d'orthodoxie communiste - mais de les éduquer à la liberté, et de ne plus faire fonctionner la relation éducative sur la contrainte et la punition. Pour cela, on souhaite intégrer des activités productives et collectives dans l'éducation, un rapport étroit à la vie réelle, à l'environnement naturel et social. ${ }^{42}$

C'est cette orientation, et ce type de mouvement, qui ont impressionnés Freinet, et sur lesquels il attire l'attention de ses lecteurs. Cela ne signifie pas que tout soit parfait en Russie : «nous répétons encore une fois que nous savons combien l'organisation russe est loin de l'idéal $\aleph^{43}$. Car précisément - et Freinet le repère dans des articles où il remarque que l'effort de transformation pédagogique révolutionnaire est porté aussi par des gens qui, sans être contre-révolutionnaires, ne sont pas des révolutionnaires patentés ou des tenants de toujours des pédagogies nouvelles ${ }^{44}$ - précisément donc, le mode opératoire révolutionnaire lui-même, en tant qu'il est ancré dans le mouvement des masses, et donc dans la multiplicité des initiatives, subordonné à leur contingence et à leur indétermination, à leur pluralité relativement éparse et à leur tâtonnement, constitue à la fois le ressort et le frein du processus de transformation - c'est bien en cela qu'il s'agit d'un « effort».

\section{9}

C'est pourquoi, quand Freinet soutient qu'il faudrait transformer la société pour espérer faire quelque chose comme une éducation nouvelle ${ }^{45}$, il indique moins l'horizon d'une solution qu'il ne pointe un problème. En appelant les pédagogues à considérer l'articulation intime qui rapporte l'école à la société, Freinet plaide en effet moins pour subordonner la révolution pédagogique ou scolaire à la révolution sociale, qu'il ne s'efforce de poser les termes du problème qu'elles posent l'une comme l'autre : il ne peut s'agir, ni à l'école ni dans la société, de fantasmer le processus révolutionnaire comme un événement, fait en un coup, et il convient de prendre la mesure de la difficulté et des résistances sans lesquelles il n'est pas de pratique révolutionnaire, au sens où celle-ci renvoie non pas à la recréation imaginaire d'un monde dans le monde ou à la place du monde fantasmatiquement mis entre parenthèses et magiquement annulé, mais à une pratique de transformation lente, difficile, une épreuve du réel, dont il faut peut-être aller jusqu'à dire qu'elle n'est mise en oeuvre que parce qu'elle est ou qu'elle semble, paradoxalement, d'abord impossible.

Témoin cet article publié par Freinet en janvier 1929 dans L'Ecole émancipée, « Réalisateurs révolutionnaires » :

A nous, révolutionnaires, de profiter d'abord des déclarations démocratiques et humanitaires de nos maîtres - comme les ouvriers ont profité au maximum des lois bourgeoises pour s'organiser révolutionnairement - afin de porter l'école, en régime capitaliste, au maximum possible de développement.

Ce maximum varie d'ailleurs avec la constitution économique et politique de chaque pays; mais il y a, dans tous les états bourgeois, un degré d'évolution de l'école qui ne peut être dépassé; celui au-delà duquel l'éducation menace les privilèges de classe. De sorte que notre lutte pour l'amélioration pédagogique et scolaire restera toujours une lutte révolutionnaire, qu'elle ait pour but d'arracher à l'état bourgeois des améliorations pourtant normales, ou qu'elle s'applique à 
démasquer les hypocrites démocrates qui parlent inconsidérément d'éducation du peuple.

(...)

Mais, et ce doit être aussi une autre originalité, nous ne nous faisons aucune illusion sur le résultat de nos efforts. Nous savons combien une tâche scolaire ainsi conçue est ingrate: nous ne sommes pas toujours compris des parents eux-mêmes: les réactionnaires de tous poils, et leurs soutiens les cléricaux, nos chefs parfois, hélas ! se dressent inévitablement sur notre chemin.

Même réussirions-nous totalement dans notre rayon primaire, n'avons-nous pas contre nous toute la société actuelle qui, loin de poursuivre et de compléter l'oeuvre d'éducation que nous avons commencée, s'applique à son action obscurantiste et destructrice : le cinéma, le théâtre, les livres, la presse... l'église, l'armée, auront beau jeu d'étouffer la bonne graine que nous essayons de faire germer.

Nous continuerons pourtant : si nous ne poursuivons pas, dans le cadre de la société actuelle, une impossible libération, nous avons cependant confiance dans le "gouffre du peuple». Nous savons que notre action ne sera point perdue. En attendant qu'un régime libérateur vienne, par son organisation au service $d u$ peuple, amplifier notre modeste effort éducatif, nous aurons du moins la satisfaction d'élargir dès maintenant l'horizon prolétarien et d'éveiller le sens de classe, en faisant sentir aux plus humbles les possibilités immenses d'une éducation créatrice.

Nous n'oublions pas enfin que l'étude attentive du problème éducatif et la participation à notre effort pour l'amélioration scolaire font comprendre à ceux qui s'y adonnent la réalité capitaliste et les nécessités révolutionnaires. L'insuccès même de leurs efforts est pour eux un enseignement qui vient affermir chaque jour davantage leur croyance à la nécessité de la lutte politique et sociale pour l'avènement d'une société d'essence éducatrice. ${ }^{46}$

Ce n'est plus, ici, les difficultés de l'action révolutionnaire qui font apparaitre nécessaire une transformation radicale de la relation pédagogique. C'est l'inverse: ce sont les difficultés de la transformation pédagogique, ce sont ses échecs qui doivent faire apparaitre d'autant plus nécessaire la transformation radicale du monde social. C'est dans cette double impasse que se situe la démarche de Freinet, en tant que démarche divisée, clivée entre deux plans, pédagogique et politique, et qui du sein même de leur écart, de leur distance, du sein même de leur différence, permet de comprendre la nécessité de leur mise en analogie à l'état pratique, pour conquérir le ressort de l'action révolutionnaire. Disons-le autrement.

L'intérêt, pour Freinet, de la transformation du rapport pédagogique, ce n'est pas d'offrir une solution pour la transformation du monde, mais d'ouvrir à des rapports sociaux nouveaux, tendant vers la réalisation intégrale des capacités humaines, susceptibles de faire relais dans le champ social quand sa transformation apparait, de son côté, bloquée ou en péril. Et inversement, la transformation des rapports sociaux doit permettre de faire relais et d'appuyer les transformations du champ pédagogique qui, sinon, sont vouées à échouer à réaliser leur propre objectif d'un plein épanouissement des facultés des individus. Ce jeu de relais, c'est précisément ce que Freinet a mobilisé pour son compte, au sein de sa classe.

\section{0}

On sait aujourd'hui que Célestin Freinet a difficilement vécu ses premières années d'instituteur, au sortir de la guerre. Pas seulement, comme l'avait indiqué Elise Freinet, 
en raison de sa blessure de guerre, qui l'aurait empêché d'assurer la classe de manière traditionnelle - pour ainsi dire, de l'extérieur de son propre désir d'instituteur. C'est plutôt ce désir lui-même que la guerre aura rendu problématique. Dans un livre récent ${ }^{47}$, Emmanuel Saint-Fuscien avance ainsi que Freinet, comme d'autres enseignants allemands et français passés sur le champ de bataille, a vécu intimement la mise en crise de l'autorité traditionnelle provoquée par la guerre, au point de chercher à changer de métier, sur le conseil d'un inspecteur qui constate ses difficultés à enseigner à Bar-surLoup, le 12 mai 1920 :

Monsieur Freinet a pour excuse un état de santé débile, résultat d'une grave blessure de guerre; mais sa classe en va pas: la discipline est faible et l'enseignement d'après la préparation n'est pas du tout approprié. Les élèves n'ont pas fait les progrès normaux. [...]

Allons! M. Freinet doit reconnaître que nous avons fait ce que nous avons pu pour lui trouver un poste qui lui convienne. J'attendais des résultats dans cette classe peu pénible. Qu'il se demande lui-même s'il a fait vraiment ce qu'il a pu : qu'il voit aussi si la profession lui convient ou non. ${ }^{48}$

"C'est pour ainsi dire une catastrophe ", commente Saint-Fuscien, qui conduit Freinet à tenter de passer le concours d'inspection, après avoir multiplié les arrêts maladie et les demandes de mutation : «à sa façon, la rentrée de cet ancien combattant mutilé à $70 \%$ reflète assez bien les difficultés ressenties par toute une génération de jeunes maîtres éprouvés. Freinet n'y est pas. Sa guerre s'impose encore à lui $»^{49}$.

L'imprimerie à l'école, et avec elle la correspondance interscolaire et la rédaction libre changeront en profondeur cette situation catastrophique, pour donner à Freinet autorité dans sa classe et en dehors, y compris, dans un premier temps, au sein même de l'Education nationale ${ }^{50}$. Mais l'introduction de l'imprimerie dans la classe de Bar-sur-Loup a lieu à la rentrée 1924 : quatre longue années séparent ainsi la première inspection de cette planche de salut. Saint-Fuscien suggère que Freinet trouve, au cours de ces années, «dans la lecture et l'écriture un moyen de survie $~_{51}$. Ne faut-il pas faire un pas de plus, et dire que, d'une part, c'est dans la lecture et l'écriture engagées, embarquées dans la lutte syndicale, que Freinet trouve son moyen de survie, et, d'autre part, qu'il s'agit, plus que d'un moyen de survie, d'un outil de transformation de sa propre vie d'instituteur, c'est-àdire de la classe et des relations qui s'y nouent? Voilà ce que Freinet conquiert sur le terrain syndical ${ }^{52}$. En effet, si Freinet lit et écrit beaucoup, publiant ses souvenirs de guerre, il se lance aussi dans un intense travail d'écriture pour le journal du syndicat, L'école émancipée - articles militants, prises de position politiques et pédagogiques, compte-rendus et notes de lectures, plus ou moins enthousiastes, plus ou moins critiques, d'ouvrages venus du courant de la pédagogie nouvelle. La lutte et l'activité de publication constituent plus que des moyens de supporter la difficulté du métier d'instituteur; elles supposent et mettent en place des collectifs vivants, des communautés de militants et de lecteurs qui, pour Freinet, ont joué un rôle d'autorisation - autorisation d'avoir quelque chose à dire et à écrire, autorisation de publier et d'être lu, autorisation de répondre dont on peut penser qu'il a non seulement déclenché un autre rapport à soi, aux autres et au monde chez Freinet, mais aussi offert, consciemment ou non, un certain nombre de dispositifs et de schèmes relationnels émancipateurs, en ce sens qu'ils autorisent à conquérir et à exercer une faculté ou une puissance. Faut-il dès lors s'étonner que ce que Freinet introduise dans sa classe un matin de l'automne 1924, ce soit une presse manuelle, la correspondance, et la rédaction libre? 
On oublie souvent que l'introduction de l'imprimerie à Bar-sur-Loup s'inscrit dans le cadre d'une critique de l'instruction scolaire qu'on aurait tort de réduire à la dénonciation du «transmissif » et du trop plein de savoirs au nom du développement de l'enfant et de sa formation morale, affective et intellectuelle. Il est frappant que, pour Freinet, cette critique de l'instruction scolaire se cristallise dans la critique des manuels ${ }^{53}$, pas seulement, parce qu'ils sont à la fois trop «chargés» de savoir et pas assez " vivants », mais aussi et surtout parce qu'ils inculquent un certain rapport au savoir que Freinet juge politiquement désastreux.

Les manuels sont un moyen d'abrutissement. Ils servent, bassement parfois, les programmes officiels. Quelques-uns les aggravent même par je ne sais quelle folie de bourrage à outrance. Mais rarement les manuels sont faits pour l'enfant. Ils déclarent faciliter, ordonner le travail du maitre; ils se vantent de suivre pas à pas... les programmes. Mais l'enfant suivra, s'il peut. Ce n'est pas de lui qu'on s'est occupé. C'est pourquoi les manuels préparent la plupart du temps l'asservissement de l'enfant à l'adulte, et plus spécialement à la classe qui, par les programmes et les crédits, dispose de l'enseignement.

[...]

Même les manuels seraient-ils bons, il y aurait tout intérêt à en réduire le plus possible l'emploi. Car le manuel, surtout employé dès l'enfance, contribue à inculquer l'idolâtrie de l'écriture imprimée. Le livre est bientôt un monde à part, quelque chose d'un peu divin, dont on hésite toujours à contester les assertions. «C'est dans le livre... » Tandis qu'il serait désirable justement d'enseigner que le livre n'est qu'une pensée imprimée - comme toute pensée sujette à l'erreur - et qu'on doit pouvoir contredire comme on contredit quelqu'un qui parle.

Les manuels tuent ainsi tout sens critique ; et c'est probablement à eux que nous devons ces générations de demi-illettrés qui croient, mot pour mot, tout ce que contient leur journal..$^{54}$

41 Et nous ajouterions aujourd'hui, de notre côté : ou qui ne croient qu'à ceux qui dénoncent systématiquement tout ce qui est donné par le biais des institutions faisant autorité école, institutions scientifiques, médias, etc. (les «complotistes», comme on dit). Précisément, ces générations de demi-illettrés qui se définissent par un rapport au savoir qui est fondamentalement et paradoxalement un rapport de croyance, dépossédés de tout moyen d'en mesurer la légitimité et la pertinence. C'est en ce sens que Charlotte Nordmann, sans mentionner Freinet, reprend aujourd'hui sa critique des manuels :

Le discours développé par les manuels scolaires, comme souvent celui des professeurs, se présente comme un discours de vérité, ou plutôt comme une vérité bien particulière, puisqu'elle ne se dégage jamais de la controverse et de la polémique, mais s'impose d'elle-même, sans jamais avoir besoin de justification.

Il est piquant de voir l'Ecole revendiquer l'héritage de ceux qui contestèrent les "autorités", quand elle-même ne cesse d'exiger de l'élève qu'il accepte par principe tout ce qui émane d'elle. L'inquiétude de nombre de professeurs à l'égard d'Internet - qui fournit matière à nombre d'« antisèches » - ne se comprend que si l'on voit que le professeur lui-même omet le plus souvent de mentionner ses sources et ne procède à aucun travail explicite sur les fondements de son discours, qui pourrait permettre à chacun de juger sa valeur. Au lieu de déplorer la naïveté des élèves, qui seraient prêts à accepter tout ce qu'ils voient affirmé avec assez d'aplomb, il conviendrait de les former à l'attention critique nécessaire pour discriminer entre les discours "fondés» et ceux qui ne le sont pas. L'Ecole, aujourd'hui, se dispense de le faire..$^{55}$ 
42 manuels présentent un savoir tout fait, pour ainsi dire sans auteur, un savoir en soi, comme s'il n'avait jamais été produit dans des conditions sociales, matérielles, techniques déterminées, et en fonction de conflits épistémologiques qui en relativisent moins la valeur qu'ils ne lui donnent, à l'inverse, la juste portée de sa légitimité : car à se présenter comme l'évidence du réel, un tel savoir est aussi facilement rejeté qu'il n'est béatement accepté, impliquant, dans cette «idolâtrie de l'écriture imprimée », la même dépendance et passivité à l'égard de ce qui à la fois semble faire autorité et s'avère absolument fragile.

43 C'est pour répondre - non pour résoudre, mais pour répondre - à ce problème que Freinet tente l'introduction de l'imprimerie à l'école. Non pas une nouvelle école, un plan global de rééducation, la création d'un nouveau milieu adéquat à l'enfant, non: l'imprimerie, solution à un problème précis posé par la réalité de la classe rurale telle qu'elle est donnée dans les conditions de l'école bourgeoise, qui impose l'apprentissage de la lecture et de l'écriture des caractères imprimés, sous une modalité abrutissante, à savoir celle de la vénération a-critique pour la lettre. En s'attaquant aux manuels, et en cherchant une autre technique d'apprentissage, Freinet s'en prend à l'une des modalités spécifiquement scolaires de la domination, moins pour l'annuler purement et simplement par le moyen d'un outil qui serait « en lui-même » emprunt de communisme, que pour la faire apparaître dans l'écart même qui s'en sépare et qui en esquisse une alternative limitée et précaire. En ce sens, l'introduction de l'imprimerie à l'école, et l'effort fait pour l'utiliser dans une perspective de coopération et d'activité, ne sont jamais acquis, parce qu'ils dépendent des situations singulières de chaque classe. Autrement dit, il faut sans cesse remettre l'ouvrage sur le métier au sein de la classe. ${ }^{56}$

\section{2}

C'est en octobre 1924 que Freinet apporte une petite presse manuelle dans sa classe. Il en rend compte une première fois au mois de juin 1925 pour la revue Clarté. Cet article inscrit l'introduction de l'imprimerie dans le cadre du problème typique de la pédagogie nouvelle : rendre l'école vivante, rompre avec les tâches spécifiquement scolaires jugées factices, inutiles et sans intérêt, avec les tâches requises par la vie elle-même. Freinet montre ainsi comment l'imprimerie à l'école permet aux élèves de s'emparer de n'importe quel événement imposé par le hasard de la vie et d'en faire l'objet d'un récit que l'on imprime et que l'on partage avec les autres. Ainsi l'apprentissage de l'écriture et de la lecture peut-il être ancré dans la mobilité même de la vie, inanticipable pour le maître.

Un bon maître peut organiser la succession des leçons et des devoirs de façon à s'appuyer constamment sur l'intérêt dominant et les besoins spontanés de sa classe. Mais sur quels textes imprimés fera-t-il l'apprentissage de la lecture qui demande pourtant un effort incessant? Comment choisira-t-il les livres?

Il prendra un des récents manuels où les diverses leçons sont soigneusement réparties selon un ingénieux système de centres d'intérêt. ${ }^{57}[. .$.

Mais qui donc cataloguera, qui aura la prétention d'immobiliser dans un livre une vie aussi mobile, et aussi diverse selon les régions que celles de nos petits écoliers?

On voit l'avantage de l'imprimerie: l'apprentissage de la lecture et de l'écriture imprimées peut s'articuler à la vie et à ses variations propres. L'imprimerie ouvre ainsi à la production d'un livre de vie, reprenant l'ensemble des textes imprimés par les élèves, 
qui soit spécifique à la singularité de la vie de chaque classe, composé au jour le jour, en fonction des intérêts des enfants, et qui peut ainsi remplacer les manuels. Mais voit aussi, par là même, à quel point Freinet reprend et tient compte de l'exigence constitutive de l'école élémentaire de la Troisième République. C'est dire que l'ouverture de l'école sur la vie ne relève pas, chez le jeune Freinet du moins, de l'utopie de l'abolition de l'école. Freinet peut bien avoir, comme à l'horizon, la perspective d'une société sans école devenue éducatrice ; mais une chose est d'en faire un horizon régulateur, autre chose est de croire en sa possibilité ici et maintenant et de vouloir sa réalisation sans délai. Avec l'imprimerie, Freinet assume la tâche qui lui est fixée par l'Education nationale, mais en travaillant à sa transformation, et ainsi en modulant l'horizon communiste qui est le sien en fonction des conditions déterminées de la III ${ }^{e}$ République : il s'agit moins de supprimer l'école que d'en faire "une manifestation plus riche et plus intense de la vie $"^{59}$, autant dire se confronter, en régime capitaliste, à l'impossibilité de ce projet, et par conséquent éprouver la nécessité de coupler cette révolution scolaire impossible à la lutte sociale et politique révolutionnaire

\section{NOTES}

1. Voir Françoise Mayeur, Histoire de l'enseignement et de l'éducation III. 1789-1930, Paris, Perrin, Tempus, 2004.

2. Fondé à Genève, inscrit au secrétariat de la Société des Nations, le BIEN avait "pour but d'établir des rapports d'entr'aide scientifique entre les différentes Ecoles nouvelles, de centraliser les documents qui les concernent et de mettre en valeur les expériences psychologiques faites dans ces laboratoires de la pédagogie de l'avenir » (Pour l'ère nouvelle, $\mathrm{n}^{\circ} 1$, 1922).

3. Fondée lors du Congrès de Calais, la LIEN est rattachée au BIEN, et met l'accent sur le respect de l'individualité de l'enfant, l'attention aux différentes formes d'activité (intellectuelle, esthétique, sociale, morale, affective, physique, etc.), le souci d'une éducation adaptée à chaque âge de la vie, la mise en oeuvre d'une coopération entre les élèves, la réflexion sur la «coéducation des sexes» et l'horizon humaniste de l'éducation. Elle prévoit une coopération renforcée entre les écoles nouvelles et acteurs de l'éducation nouvelle notamment par l'organisation de congrès internationaux bisannuels. (Pour l'ère nouvelle, $n^{\circ} 1,1922$ ). Selon Laurent Gutierrez, la LIEN visait moins à rompre avec un modèle traditionnel périmé qu'à coordonner des alternatives à ce modèle vécues comme des expérimentations pédagogiques destinées à réformer le monde scolaire sans rejeter pour autant « un modèle qui a fait ses preuves » (Laurent Gutierrez, «La ligue internationale pour l'éducation nouvelle. Contribution à l'histoire d'un mouvement international de réforme de l'enseignement, dans Les Sciences de l'Education. Pour l'ère nouvelle, $n^{\circ} 45,2009$ ). Il convient en effet de rappeler que ce mouvement, pour s'appuyer sur des initiatives privées qui peuvent plus facilement s'autoriser des formes originales d'apprentissage et d'éducation, n'en était pas moins porté également par des pédagogues de l'enseignement public ou soucieux de le défendre, comme Roger Cousinet par exemple, mais aussi Ferdinand Buisson qui y apporte sa caution morale. 
Sur le Congrès fondateur de Calais, qui s'est tenu du 30 juillet au 12 août 1921, voir Béatrice Ensor, « Notre premier Congrès International d'Education nouvelle », dans Pour l'ère nouvelle, $\mathrm{n}^{\circ} 1$, 1922.

4. L'ensemble des numéros de Pour l'ère nouvelle est disponible en ligne sous forme de pdf sur le site de l'Université de Caen : http://www.unicaen.fr/recherche/mrsh/pen La revue est publiée jusqu'en 1940, interrompue pendant la guerre puis reprise en 1946 et 1947, avant de changer de nom et de devenir la revue des Sciences de l'éducation - pour l'ère nouvelle, qui existe encore aujourd'hui aux presses universitaires de Caen.

5. Voir Emmanuel Saint-Fuscien, Célestin Freinet. Un pédagogue en guerres : 1914-1945, Paris, Perrin, première partie. Freinet publiera en 1921 un récit de son expérience de guerre, sous le titre Touché! Souvenirs d'un blessé de guerre.

6. Il est déclaré invalide à $70 \%$.

7. Cf. les nombreux compte-rendus qu'il donne de ses lectures dans la revue L'Ecole émancipée dès le tout début des années 1920.

8. Il s'agit de Pierre Bovet, philosophe et psychologue suisse, appelé par Claparède lorsqu'il fonde l'Institut Jean-Jacques Rousseau de Genève en 1912 pour en assurer la direction, ce qu'il fera jusqu'en 1944 (avec Piaget à partir de 1933). Voir la notice «Institut Jean-Jacques Rousseau » rédigée par Daniel Hameline pour le Dictionnaire historique de la Suisse, en ligne: http:// www.hls-dhs-dss.ch/textes/f/F10431.php

9. Célestin Freinet, «Vers l'école du prolétariat », Clarté n 47, 15 novembre 1923, p. 455-456.

10. Grégory Chambat suggère que « l'incompréhension qui va s'installer, à la fin des années 1930 , entre Célestin Freinet et le syndicalisme enseignant a contribué à renforcer la légende d'un modeste instituteur "contraint" à l'innovation pédagogique du fait de son état de santé.» (Grégory Chambat, L'école des barricades. Vingt-cinq textes pour une autre école, 1789-2014, Paris, Libertalia, p. 113). Henri Peyronie remarque que cette explication de la recherche par Freinet de nouvelles méthodes pédagogiques, également privilégiée par Elise Freinet, a sans doute été retenue par la légende, "par exemple dans les Ecoles normales d'instituteurs, parce qu'elle euphémisait la dimension politique radicale de l'engagement de Freinet. » Henri Peyronie, Le mouvement Freinet : du fondateur charismatique à l'intellectuel collectif, Caen, Presses universitaires de Caen, 2013, p. 22.

11. Voir Laurence Biberfeld et Grégory Chambat, Apprendre à désobéir : petite histoire de l'école qui résiste, Paris, Libertalia, 2013, p. 57 et suivantes, ainsi que l'édition par Catherine Chambrun et Grégory Chambat de Célestin Freinet, Le maître insurgé. Articles et éditoriaux 1920-1939, Paris, Libertalia, 2016.

12. Célestin Freinet, « Vers l'école du prolétariat », Clarté $n^{\circ} 47$, novembre 1923, op. cit.

13. On peut ainsi reprendre l'explication avancée par l'historien de l'enseignement Antoine Prost à propos de la poussée contestataire des années 1920 : les "démobilisés les plus mécontents » auraient été attirés vers « les syndicats franchement contestataires, voire révolutionnaires. » (cf. Emmanuel Saint-Fuscien, Célestin Freinet. Un pédagogue en guerres : 1914-1945, Paris, Perrin, p. 76).

14. Célestin Freinet, « Contre une pédagogie syndicale », L'Ecole émancipée, 4 juin 1921.

15. Célestin Freinet, « Pour la Révolution à l'Ecole », L'Ecole émancipée, 12 mars 1921.

16. Par exemple dans le numéro de juillet 1933 de L'éducateur prolétarien, la revue de la Coopérative de l'enseignement laïque (C.E.L.) qui succède au bulletin L'imprimerie à l'école à partir de la rentrée (octobre) 1932, quand Freinet présente son projet d'école nouvelle en dehors de l'éducation nationale après "l'affaire Freinet »: «Nous voudrions, de plus, tenter une grande expérience qui, par-delà l'évolution de nos techniques, influencerait certainement toute la pédagogie : en matérialistes convaincus, nous voudrions recréer cette pédagogie, penser au corps avant de torturer l'esprit, agir sur le milieu avant de vouloir façonner les individus; mettre pratiquement les enfants dans des conditions physiques et économiques susceptibles d'améliorer et de libérer leur corps ; organiser ensuite leur vie de façon à conserver intact cet enthousiasme 
créateur dont nous avons fait un des pivots de notre pédagogie; alimenter cet enthousiasme pour que nos élèves se saisissent vigoureusement de la civilisation ambiante et bâtissent ainsi leur part d'avenir. "

17. Voir en particulier la section qu'Elise Freinet y consacre dans Naissance d'une pédagogie populaire, op. cit., p. 44-46.

18. Alain Vergnioux, Cinq études sur Célestin Freinet, Caen, Presses universitaires de Caen, 2005, p. 31

19. Ibid., p. 29.

20. Célestin Freinet, «L'Ecole du Travail », dans Clarté $\mathrm{n}^{\circ} 62,1^{\mathrm{er}}$ juillet 1924.

21. Célestin Freinet, «Vers l'école du prolétariat. La dernière étape de l'école capitaliste », Clarté $n^{\circ} 60,1^{\text {er }}$ juin 1924.

22. Freinet se rend près de Hambourg à l'été 1922 et rend compte de l'école qu'il y a visitée, en compagnie d'Heinrich Siems, dans « Une visité dans une école prussienne », paru dans les $n^{\circ} 5$ et 6 d'octobre et novembre 1922 de L'Ecole Emancipée.

23. Ibid.

24. D’où la méfiance de Freinet, en 1932, face au succès rencontré par la « coopération scolaire » de M. Profit. Voir Elise Freinet, Naissance d'une pédagogie populaire, p. 158-159.

25. Célestin Freinet, « Note de pédagogie nouvelle révolutionnaire ", L'Ecole émancipée, n 10, 27 novembre 1927.

26. Marx et Engels, Manifeste du parti communiste, tr. M. Rubel et L. Evrard, dans Karl Mars, Oeuvres . Economie I, Gallimard, Pléiade, 1963, p. 191-192.

27. Célestin Freinet, « La discipline nouvelle. Quelques réalisations », art. cit.

28. Célestin Freinet, «Mes impressions de pédagogue en Russie soviétique : le travail et la vie à l'école russe ", L'Ecole émancipée, $\mathrm{n}^{\circ}$ 13, 20 décembre 1925.

29. Célestin Freinet, « Vers l'école du prolétariat », Clarté n 47, 15 novembre 1923.

30. Célestin Freinet, «La discipline nouvelle. Quelques réalisations », Clarté $n^{\circ} 49,15$ décembre 1923.

31. Au point que, dans un livre récent, on a pu considérer que la vision que Freinet défend de l'URSS, et en particulier des initiatives pédagogiques, était troublée par « des lunettes teintées de rose » qui l'inclinaient non seulement à commettre des erreurs, mais à " persister » et à tenter de les «justifier». Carole Hardouin Thouard et Alexeï Ovtcharenko, Célestin Freinet en URSS (1925): "Le pédagogue et l'écrivain », Paris, L'Harmattan, 2017.

32. Célestin Freinet, «Mes impressions de pédagogue en Russie soviétique : le travail et la vie à l'école russe ", L'Ecole émancipée, n 13, 20 décembre 1925.

33. Ibid.

34. Pour laquelle il publiera à l'été 1926 deux articles - les deux premiers, dans cette revue présentant «L'effort pédagogique russe ». Voir Pour l'ère nouvelle, $\mathrm{n}^{\circ} 21$ et $\mathrm{n}^{\circ} 22$, respectivement parus en juillet et en septembre 1926. Delaunay les annonce d'ailleurs dès décembre 1925 dans les objections qu'il adresse à Freinet. En janvier 1928, dans un texte qui présente, de manière critique mais non dénuée d'intérêt, les numéros de Pour l'ère nouvelle consacrés au Congrès de Locarno de la LIEN, Freinet émet un jugement expéditif des plus sévères sur ces deux textes de Delaunay : «Comment [...], depuis dix ans se développe là-bas [en Russie] une expérience aussi impressionnante de rénovation scolaire, un expérience qui dépasse de beaucoup tout ce qui a pu se faire dans le monde en ce début de siècle pour améliorer l'éducation populaire et la Ligue pour l'éducation nouvelle, la revue Pour l'ère nouvelle ne nous en ont rien dit ? - Pardon, s'excusera M. Ferrière : il y a deux articles de Delaunay ! Quelle que soit la documentation de camarade, ce qu'il a dit de l'éducation russe est ridiculement insuffisant. » Célestin Freinet, «Le Congrès de Locarno (suite) », L'Ecole émancipée, n 19, 29 janvier 1928.

35. «Mais si les programmes russes et les instructions méthodologiques qui les accompagnent présentent une réelle supériorité sur les programmes officiels de France et de Belgique, il ne 
s'ensuit pas que nous devions les considérer comme un tout parfait qu'il n'y a nul besoin de compléter ou de corriger.» E. Delaunay, «Pédagogie russe et pédagogie bourgeoise ", L'Ecole Emancipée, $\mathrm{n}^{\circ}$ 12, 13 décembre 1925.

36. Ibid.

37. C'était par ailleurs indéniable. Concernant l'influence décisive de la pédagogie de Dewey en particulier sur les expériences pédagogiques russes des années 1920, voir Guillaume Garetta, "L'école en révolution. L'application des méthodes deweyennes en Russie soviétique », dans Denis Kambouchner et François Jacquet-Francillon (éd.), La crise de la culture scolaire, Paris, PUF, 2005, 141-158.

38. Célestin Freinet, « Pédagogie russe et pédagogie bourgeoise ", L'Ecole émancipée, $\mathrm{n}^{\circ} 15$, janvier 1926.

39. Voir Nicolas Werth, "Alphabétisation et idéologie en Russie soviétique ", dans Vingtième Siècle, revue d'histoire, $\mathrm{n}^{\circ}$ 10, avril-juin 1986.

40. Oleg Bernaz, «Développement psychique et apprentissage scolaire: Lev Vygotski et le problème de l'éducation », dans Les Carnets du Centre de Philosophie du Droit, n 161, 2013.

41. Mais Nicolas Werth remarque que, au même moment, "l'alphabétisation marque le pas", art. cit., p. 28.

42. Guillaume Garetta, «L'école en révolution », art. cit.

43. Célestin Freinet, «La Russie révolutionnaire est notre patrie pédagogique », L’Ecole émancipée, $\mathrm{n}^{\circ}$ 7, 6 novembre 1927.

44. "Dans une classe de 4e année, aussi nombreuse - garçons et filles naturellement - nous tombons en plein travail d'organisation scolaire du self-government. L'institutrice n'a pas l'air ni novatrice, ni bien révolutionnaire. C'est une humble petite femme grisonnante, déjà ancienne dans le métier sans doute, et que la vie a débordée. Car l'élan des jeunes et les institutions nouvelles sont parfois plus forts que toutes les résistances "raisonnables" » Célestin Freinet, "Mes impressions de pédagogue en Russie soviétique : une école ordinaire du 1er degré », L'Ecole émancipée, $\mathrm{n}^{\circ} 10,29$ novembre 1925.

45. «Non, l'école à elle seule, et malgré ses efforts, ne bâtira pas la société nouvelle. Ferrière luimême l'a dit : il ne suffit pas de réformer l'école; il faut la transformer. Nous ne croyons pas, nous, cette révolution possible à l'école au milieu d'une société contre-révolutionnaire. Il serait donc vain d'attendre de l'école une modification lente et pacifique des esprits, et qui serait capable de faire faire l'économie d'une révolution. » Célestin Freinet, "Mes impressions de pédagogue en Russie soviétique : le travail et la vie à l'école russe », art. cit.

46. Célestin Freinet, « Réalisateurs révolutionnaires ", L'Ecole émancipée n 18, 27 janvier 1929.

47. Emmanuel Saint-Fuscien, Célestin Freinet. Un pédagogue en guerres 1914-1945, op. cit.

48. «Bulletin d'inspection du 12 mai 1920 », cité dans Emmanuel Saint-Fuscien, Célestin Freinet. Un pédagogue en guerres, op. cit., p. 73.

49. Ibid., p. 74.

50. Emmanuel Saint-Fuscien le souligne : «Cette "idée fondatrice" [l'introduction de la presse en classe] bénéficia d'une double bienveillance. Par son aspect innovant et collectif, par sa volonté de tourner le dos aux manuels scolaires, elle séduit rapidement un nombre important de pédagogues rattachés à l'éducation nouvelle. Mais aussi, par la rigueur qu'implique cette méthode mobilisant des exigences scolaires traditionnelles comme la rédaction, l'écriture, l'orthographe, la grammaire ou la ponctuation, elle apparaît également compatible avec "les pratiques canoniques de l'école primaire française". Les bulletins d'inspections de Freinet entre 1924 et 1929 en témoignent sans ambiguïé : l'initiative est appréciée par les autorités académiques. » Ibid., p. 78.

51. Ibid.

52. Je tiens ici à remercier les collègues du laboratoire Logiques de l'agir, de l'Université de Franche-Comté (Besançon), pour leurs remarques et suggestions à l'égard desquelles les lignes 
qui suivent sont redevables: Vincent Bourdeau, Arnaud Macé, Julien Pasteur, Carole Widmaier et, en particulier, Sophie Audidière.

53. Le tout premier texte qui annonce l'imprimerie à l'école, sans encore développer les modalités et les conséquences de cette technique, porte sur «Les manuels scolaires » (Clarté $\mathrm{n}$ -73, avril 1924).

54. Ibid. On ne peut s'empêcher de penser ici aux analyses de Renée Balibar et Dominique Laporte dans Le français national (Renée Balibar et Dominique Laporte, Le français national. Politique et pratique de la langue sous la Révolution française, Paris, Hachette, 1974).

Balibar et Laporte y montrent - en s'attachant en particulier à l'enseignement de la langue nationale - que la construction de l'instruction scolaire par la bourgeoisie révolutionnaire et post-révolutionnaire comme instruction élémentaire institue un rapport de dépossession et d'incompréhension relative des enfants à l'égard du savoir enseigné à l'école. L'argument de Balibar et Laporte, c'est que les "éléments » ou les "fondamentaux » de savoir enseignés sont abstraitement et artificiellement construits comme éléments "simples» déconnectés de leur production réelle, des problèmes qui les requièrent, et, par là, de leur signification profonde selon un processus qu'ils appellent un processus "d'élémentation ». Aussi les élèves sont non seulement dans l'impossibilité première de comprendre véritablement les savoirs enseignés, mais surtout dans l'impossibilité d'être en mesure d'y entretenir un rapport actif et critique, du moins tant qu'ils n'ont pas accès aux formes réelles, complexes et effectivement concrètes de savoir dont ces éléments ont été abstraitement tirés. Par exemple, pour la langue française : le français élémentaire artificiellement fabriqué en phrases soi-disant simples, qu'en réalité personne ne prononce, en règles grammaticales soi-disant fondamentales, en règles de conjugaison soi-disant nécessaires, mais dont le sens n'est réellement compréhensible que rapporté au latin qui en supporte la structure et dont l'apprentissage est réservé aux collèges et institution de l'enseignement secondaire que n'atteignent à peu près que le seuls enfants des classes bourgeoises.

A sa manière, c'est ce que, pour son compte, dénonçait déjà Freinet dans les manuels et, plus largement, dans la centralité de l'instruction au sein de l'école de la III ${ }^{\mathrm{e}}$ République : "Dès ses débuts, notre école nationale laïque a idolâtré l'Instruction; elle a pensé qu'enseigner les premiers éléments de la lecture, de l'écriture, des sciences, devait contribuer à l'élévation maximum des citoyens. » (Célestin Freinet, «Vers une méthode d'Education nouvelle pour les écoles populaires", dans L'imprimerie à l'Ecole, décembre 1928, cité par Elise Freinet, Naissance d'une pédagogie populaire, op. cit., p. 78).

55. Charlotte Nordmann, La fabrique de l'impuissance 2. L'école, entre domination et émancipation, Paris, Amsterdam, 2007.

56. « Oh ! je ne considère point l'imprimerie à l'Ecole comme une panacée. Je ne cesse de spécifier que l'imprimerie à l'école n'est nullement une méthode; c'est une technique, un outil, que chacun emploie comme il l'entend, au mieux des intérêts de son école. » Célestin Freinet, «La correspondance interscolaire réalisée par l'imprimerie à l'école ", L'Ecole émancipée n 38, 19 juin 1927.

57. Freinet fait ici référence au système des centres d'intérêt mis en place par Ovide Decroly en particulier, autour de deux domaines fondamentaux de connaissances auxquelles l'enfant est intéressé : la connaissance de sa propre personnalité et la connaissance du milieu dans lequel il vit.

58. Célestin Freinet, «Contre un enseignement livresque : l'imprimerie à l'école », Clarté $\mathrm{n}^{\circ} 75$, juin 1925.

59. Ibid. 


\section{RÉSUMÉS}

Ce texte met l'accent sur deux dimensions du projet théorique et pratique de Freinet, matérialisme et révolution, qui le singularisent dans le paysage des pédagogies nouvelles. Une telle perspective suppose de considérer le travail de Célestin Freinet comme un travail mené en situation (au sens de Sartre), et par conséquent de le considérer en train de se faire, dans un débat permanent avec la conjoncture théorique et pratique dans laquelle il s'élabore, sans présupposer certains de ses résultats comme évidents ou donnés d'emblée. A partir d'une relecture précise des premiers articles publiés par Freinet au cours des années 1920 pour les revues Clarté et L'école émancipée, et de son engagement syndical, il s'agit de mettre en évidence et d'éclairer la manière dont le matérialisme révolutionnaire de Freinet a, non seulement rendu possible, mais déterminé en profondeur ses premières tentatives pédagogiques, en premier lieu la fameuse « imprimerie à l'école».

\section{INDEX}

Mots-clés : Freinet, matérialisme, révolution, pédagogie nouvelle, syndicalisme enseignant, imprimerie à l'école

\section{AUTEUR}

\section{ANTOINE JANVIER}

Antoine Janvier est chercheur au Département de philosophie de l'Université de Liège (Belgique) où il enseigne la philosophie politique et la philosophie de l'éducation. Il est également membre du GRM. 